TO APPEAR IN The Astrophysical Journal Letters.

Preprint typeset using LATEX style emulateapj v. 26/01/00

\title{
THE LOW-MASS, HIGHLY ACCRETING BLACK HOLE ASSOCIATED WITH THE ACTIVE GALACTIC NUCLEUS 2XMM J123103.2+110648
}

\author{
LUIS C. Ho ${ }^{1}$, MinJIN KIM ${ }^{1,2}$, AND YUICHI TERASHIMA ${ }^{3}$ \\ To appear in The Astrophysical Journal Letters.
}

\begin{abstract}
Optical spectra and images taken with the Baade 6.5 meter Magellan telescope confirm that 2XMM J123103.2+110648, a highly variable X-ray source with an unusually soft spectrum, is indeed associated with a type 2 (narrow-line) active nucleus at a redshift of $z=0.11871$. The absence of broad $\mathrm{H} \alpha$ or $\mathrm{H} \beta$ emission in an otherwise $\mathrm{X}$-ray unabsorbed source suggests that it intrinsically lacks a broad-line region. If, as in other active galaxies, the ionized gas and stars in J1231+1106 are in approximate virial equilibrium, and the $M_{\mathrm{BH}}-\sigma_{*}$ relation holds, the exceptionally small velocity dispersion of $\sigma=33.5 \mathrm{~km} \mathrm{~s}^{-1}$ for [O III] $\lambda 5007$ implies that $M_{\mathrm{BH}} \approx 10^{5} M_{\odot}$, among the lowest ever detected. Such a low black hole mass is consistent with the general characteristics of the host, a small, low-luminosity, low-mass disk galaxy. We estimate the Eddington ratio of the black hole to be $\gtrsim 0.5$, in good agreement with expectations based on the X-ray properties of the source.
\end{abstract}

Subject headings: black hole physics — galaxies: active — galaxies: nuclei — galaxies: Seyfert

\section{INTRODUCTION}

Active galactic nuclei (AGNs) in low-mass, late-type galaxies provide important insights into the demographics of nuclear black holes (BHs) on the bottom end of the $\mathrm{BH}$ mass function. With typical masses $M_{\mathrm{BH}} \lesssim 10^{6} M_{\odot}$, this class of AGNs not only helps to illuminate accretion physics in a poorly explored regime of parameter space, but it also serves as local analogs for the seeds of supermassive BHs in quasars. To date the bulk of the known low-mass BHs have been discovered in the optical (Filippenko \& Ho 2003; Barth et al. 2004, 2008; Greene \& Ho 2004, 2007c; Ai et al. 2011), supplemented by a handful of cases found through mid-infrared spectroscopy (e.g., Satyapal et al. 2009). X-ray observations have additionally revealed a potentially important population of much more numerous lowmass BHs accreting at low rates in nearby late-type spiral galaxies (Desroches \& Ho 2009; Araya Salvo et al. 2012).

X-ray variability - a near-universal attribute of AGNspresents another potentially effective strategy to search for lowmass BHs. As both the amplitude and timescale of AGN variability depend on mass (e.g., Papadakis 2004), lower mass objects are expected to vary more strongly and more rapidly. Previous X-ray variability studies indeed support this trend (NGC 4395: Vaughan et al. 2005; POX 52: Thornton et al. 2008; SDSS-selected objects: Minniuti et al. 2009). Recently, Kamizasa et al. (2012) utilized the XMM-Newton serendipitous source catalog to identify 15 variable $X$-ray sources that are good AGN candidates. From the strength of the X-ray variability, they predict that these AGNs have $M_{\mathrm{BH}} \approx(1-7) \times 10^{6}$ $M_{\odot}$.

Terashima et al. (2012) highlight the most extreme case from Kamizasa et al.'s survey, 2XMM J123103.2+110648 (hereinafter J1231+1106 for brevity). The X-ray properties of this source are exceptional in at least two respects. Its X-ray light curve shows not only strong variability (factor of 3 or more within $1000 \mathrm{~s}$ ) but, in at least one of its three observations, possibly also quasi-periodic modulations with a period of $\sim 15,000 \mathrm{~s}$. More remarkable still is its X-ray spectrum. The spectrum of J1231+1106 completely lacks emission at energies greater than $\sim 2 \mathrm{keV}$ and can be described entirely by a soft thermal component. No significant intrinsic X-ray absorption is detected. Such an extreme soft excess is unprecedented among AGNs. Modeling the soft excess with a multicolor disk blackbody yields an inner disk temperature of $k T=0.16-0.21$ $\mathrm{keV}$. Moreover, the soft X-ray band shows spectral variability consistent with Comptonization. Both the strength of the soft excess and the possible evidence for Comptonization suggest that the source has a high Eddington ratio ( $\gtrsim 0.3)$. In combination with the observed luminosity, the $\mathrm{BH}$ may be no more massive than $\sim 10^{5} M_{\odot}$, one of the lowest ever identified in an AGN.

Interesting though they may be, the above results strongly hinge on the correct identification of J1231+1106 as an extragalactic source. Terashima et al. (2012) argue that it is unlikely to be a Galactic source. Based on its positional coincidence, they tentatively associate $\mathrm{J} 1231+1106$ with the optical counterpart SDSS J123103.24+110648.5, a small ( $r$-band isophotal diameter $\left.\sim 2^{\prime \prime}\right)$, faint $(r=20.04 \pm 0.04 \mathrm{AB}$ model mag), but rather red $(g-r=0.83 \pm 0.04 \mathrm{mag})$ galaxy with an estimated photometric redshift of $z=0.13 \pm 0.05$ according to the Seventh Data Release of the Sloan Digital Sky Survey (SDSS; Abazajian et al. 2009). Assuming a distance of $D_{L}=610 \mathrm{Mpc}$ (based on a cosmology of $H_{0}=70 \mathrm{~km} \mathrm{~s}^{-1} \mathrm{Mpc}^{-1}, \Omega_{m}=0.3$, and $\Omega_{\Lambda}=0.7$ ), the intrinsic $0.5-2 \mathrm{keV}$ luminosity is $L_{\mathrm{X}}=(1.6-3.8) \times 10^{42}$ $\mathrm{erg} \mathrm{s}^{-1}$, where the range reflects the extremes of the observed variations. If we assign all of the optical emission to starlight, the galaxy has a maximum absolute magnitude of $M_{g}=-18.1$, characteristic of dwarf galaxies and within the range of other AGNs with low-mass BHs (Greene \& Ho 2004, 2007c). However, even if the X-ray source is associated with the faint galaxy, it is unclear whether the emission comes from the galaxy nucleus or, instead, from an exceptionally powerful off-nuclear (i.e. ultraluminous or hyperluminous) X-ray source.

\footnotetext{
${ }^{1}$ The Observatories of the Carnegie Institution for Science, 813 Santa Barbara Street, Pasadena, CA 91101, USA

${ }^{2}$ Korea Astronomy and Space Science Institute, Daejeon 305-348, Republic of Korea

${ }^{3}$ Department of Physics, Ehime University, Matsuyama, Ehime 790-8577, Japan
} 

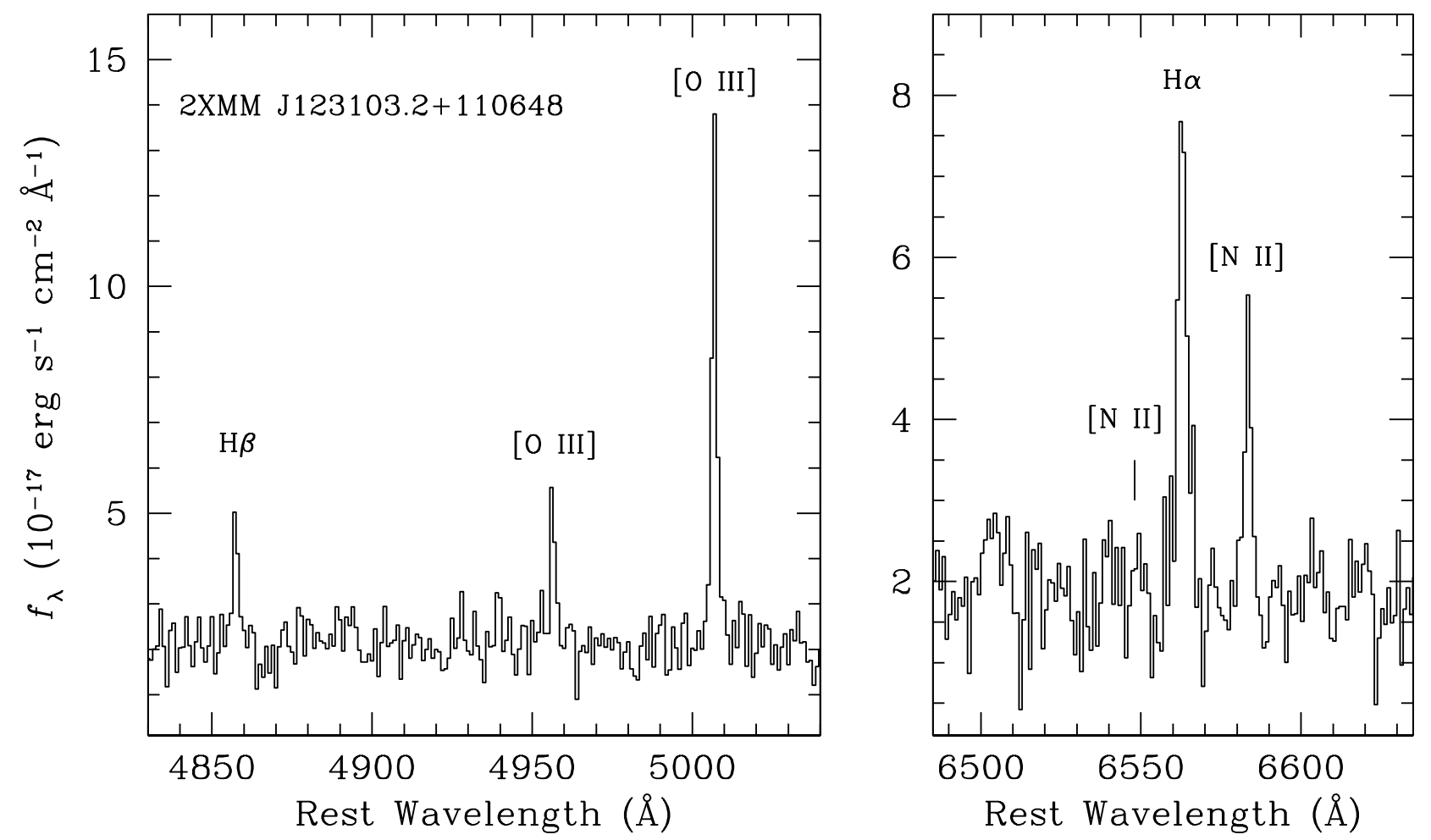

FIG. 1.- Magellan spectrum of J1231+1106, with major emission lines labeled. The blue portion of the spectrum has been scaled to match the red portion, and a redshift of $z=0.1187$ has been removed.

Using a new optical spectrum of J1231+1106, we present an accurate measurement of the redshift of this source, confirm that it is an AGN based on the excitation of its nebular lines, although no broad permitted lines are visible, and use the velocity dispersion of the narrow emission lines to constrain the mass and Eddington ratio of the central $\mathrm{BH}$.

\section{SPECTROSCOPIC OBSERVATIONS}

We observed J1231+1106 using the Inamori Magellan Areal Camera and Spectrograph (IMACS) on the Baade 6.5 meter telescope at Las Campanas Observatory. Two spectra were taken in long-slit mode with the 600 lines $\mathrm{mm}^{-1}$ grating, one on 2012 May 14 UT blazed for blue coverage (3650-6600 $⿱$ ) using a $0 . \prime 7$ slit and another on 2012 July 14 UT blazed for red coverage (5700-8900 $\AA$ ) using a 1.'2 slit. Total integration times were $3000 \mathrm{~s}$ and $1800 \mathrm{~s}$, respectively, split into multiple exposures. The sky conditions were clear and the seeing average ( 0." $\left.65-1{ }^{\prime \prime} 15\right)$. Both observations were taken at moderately high airmasses (1.4-1.6), but the slit was oriented along the parallactic angle to minimize slit losses due to differential atmospheric refraction. The instrumental resolution, estimated from the full width at half maximum (FWHM) of the night sky lines, is $1.2 \AA$ for the blue setting and $3.5 \AA$ for the red setting, which corresponds to a velocity dispersion of $\sigma_{\text {inst }}=31.8 \mathrm{~km}$ $\mathrm{s}^{-1}$ for [O III] $\lambda 5007$ and $\sigma_{\text {inst }}=68.1 \mathrm{~km} \mathrm{~s}^{-1}$ for $\mathrm{H} \alpha$.

Data reduction was performed using tasks in IRAF ing basic procedures outlined in Ho \& Kim (2009). In brief, the two-dimensional data were corrected for bias and flat-fielded using a series of external quartz lamp images. Cosmic rays were removed by combining multiple exposures using "crreject" in imcombine. The extracted one-dimensional spectra were wavelength calibrated using $\mathrm{He}+\mathrm{Ne}+\mathrm{Ar}$ arc lamp spectra and then corrected for telluric absorption and flux calibrated using spectra of the spectrophotometric standard stars Feige 56 and LTT 4816.

\section{OPTICAL PROPERTIES}

Figure 1 shows the final optical spectrum of J1231+1106. The flux density scale of the red spectrum matches closely with that expected from the SDSS photometry, confirming that our absolute spectrophotometric calibration is reasonably accurate. We measure $f_{\lambda}(6230 \AA)=(2.4 \pm 0.3) \times 10^{-17} \mathrm{erg} \mathrm{s}^{-1} \mathrm{~cm}^{-2} \AA^{-1}$; this corresponds to $r=20.17 \pm 0.13 \mathrm{AB}$ mag, to be compared to $r=20.04 \pm 0.04 \mathrm{AB}$ mag from SDSS photometry. The blue and red portions of the spectrum agree reasonably well in shape in their region of overlap $(\sim 5800-6500 \AA)$, but the absolute flux scale on the blue side is $41 \%$ lower, presumably because of the narrower slit used in the blue setting. We have accordingly scaled the blue spectrum by a factor of 1.41 .

We detect a weak continuum and sharp, narrow emission lines from $\mathrm{H} \beta$, [O III] $\lambda \lambda 4959,5007, \mathrm{H} \alpha$, and [N II] $\lambda 6583$. [N II] $\lambda 6548$ is absent, but its upper limit is consistent with $\sim 1 / 3$ of [N II] $\lambda 6583$. No broad component is seen in $\mathrm{H} \alpha$ or

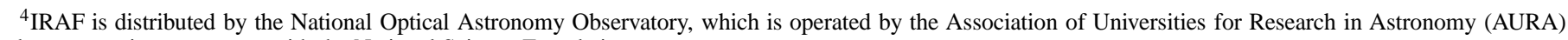
under cooperative agreement with the National Science Foundation. 


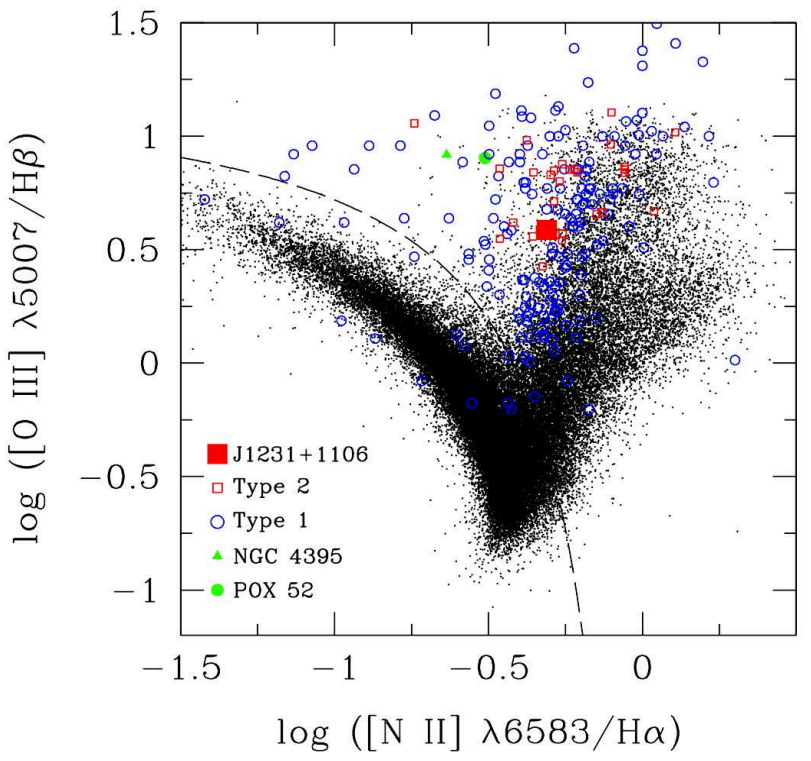

FIG. 2.- Line-ratio diagnostic diagram of [O III] $\lambda 5007 / \mathrm{H} \beta$ vs. [N II] $\lambda 6583 / \mathrm{H} \alpha$. The small black points are SDSS measurements from Kauffmann et al. (2003) with signal-to-noise ratios larger than 6. J1231+1106 is marked with a large, filled red square. Small open red squares are low-mass type 2 Seyferts from Barth et al. (2008), while the type 1 sample of Greene \& Ho (2007a) is shown as open blue circles. NGC 4395 and POX 52 (Barth et al. 2004) are plotted as a filled green triangle and circle, respectively. The dashed line represents the empirical boundary proposed by Kewley et al. (2006) to separate star-forming galaxies (left) from AGNs (right).

$\mathrm{H} \beta$. The presence of moderately strong [O III] $\lambda 5007$ emission compared to $\mathrm{H} \beta(3.86 \pm 0.04)$ and significant $[\mathrm{N}$ II] $\lambda 6583$ relative to $\mathrm{H} \alpha(0.49 \pm 0.05)$ qualifies the source as an AGN. In the diagnostic diagram of [O III] $\lambda 5007 / \mathrm{H} \beta$ vs. [N II] $\lambda 6583 / \mathrm{H} \alpha$ (Baldwin et al. 1981; Figure 2), J1231+1106 lies comfortably within the locus of high-excitation AGNs (Seyfert nuclei), but, not unlike other low-mass AGNs, it is somewhat displaced toward lower [N II] $\lambda 6583 / \mathrm{H} \alpha$, presumably because of the lower metallicity expected for the low-luminosity, low-mass host galaxy (Ludwig et al. 2012).

Our spectra barely resolve the lines in the blue but not at all in the red. The intrinsic width of [O III] $\lambda 5007$, the strongest line, is FWHM $=78.8 \pm 6 \mathrm{~km} \mathrm{~s}^{-1}$, or $\sigma=33.5 \pm 2.6 \mathrm{~km} \mathrm{~s}^{-1}$ for a Gaussian profile. This is among the narrowest emission line widths ever measured for AGNs (Barth et al. 2008; Xiao et al. 2011). The centroid of [O III] $\lambda 5007$ yields an accurate measurement of the redshift, $z=0.11871 \pm 0.000002 ; \mathrm{H} \alpha$ gives a consistent value of $z=0.1176 \pm 0.00002$, but we adopt that based on [O III] because of its higher precision. For the same set of cosmological parameters adopted by Terashima et al. (2012), the revised distance for $\mathrm{J} 1231+1106$ is $D_{L}=553 \mathrm{Mpc}$.

Assuming an intrinsic ratio of $\mathrm{H} \alpha / \mathrm{H} \beta=3.1$ for AGNs (Halpern \& Steiner 1983) and the extinction curve of Cardelli et al. (1989), the observed Balmer decrement of $\mathrm{H} \alpha / \mathrm{H} \beta=$ 3.55 corresponds to an internal extinction of $A_{V}=0.425 \mathrm{mag}$. Galactic extinction contributes an additional $A_{V}=0.092 \mathrm{mag}$ (Schlafly \& Finkbeiner 2011). Correcting for a total lineof-sight extinction of $A_{V}=0.517 \mathrm{mag}, \mathrm{J} 1231+1106$ has intrinsic line luminosities of $\left.L_{[\mathrm{O}} \mathrm{III}\right]=1.66 \times 10^{40} \mathrm{erg} \mathrm{s}^{-1}$ and $L_{\mathrm{H} \alpha}=1.32 \times 10^{40} \mathrm{erg} \mathrm{s}^{-1}$.

To gain additional insight into the nature of the host of J1231+1106, we also performed a simple analysis of the IMACS acquisition image recorded during the second observing run. The $80 \mathrm{~s} V$-band exposure is shallow, and the seeing was poor $(\mathrm{FWHM} \approx 1$.' 15$)$, but the pixel scale of the $f / 4$ camera $\left(0 .{ }^{\prime \prime} 11\right)$ does afford enough resolution to provide some rudimentary constraints on the photometric structure of the galaxy. We used the code GALFIT (Peng et al. 2010) to construct a simple two-dimensional model, which consists of a single Sérsic (1968) function convolved with a point-spread function derived from a nearby star on the same CCD chip as the galaxy. The best-fit model yields a Sérsic index of $n \approx 0.7$, close to an exponential profile $(n=1)$, and an effective radius of $R_{e}=0$." 32 $\simeq 0.7 \mathrm{kpc}$.

\section{PHYSICAL IMPLICATIONS}

We have conclusively established that the highly variable, unusually soft X-ray source J1231+1106 is of extragalactic origin at $z=0.11871 \pm 0.000002$. The optical spectrum is dominated by narrow emission lines excited by a nonstellar mechanism, presumably photoionization by the same source producing the X-rays. This confirms that J1231+1106 is indeed an AGN.

No broad $\mathrm{H} \alpha$ or $\mathrm{H} \beta$ is seen in the spectrum, making this a type 2 AGN not dissimilar from those reported by Barth et al. (2008). A key distinction, however, is that while the Barth et al. (2008) sources are highly absorbed in the X-rays (Thornton et al. 2009), J1231+1106 is not. Its XMM-Newton spectrum shows no evidence of any significant intrinsic X-ray absorption. Comparing the X-rays with the strength of the [O III] emission further supports this, as illustrated in Figure 3. For reference we show the best-fitting relation between $L_{\mathrm{X}}$ and $L_{[\mathrm{O}}$ III] for luminous, unabsorbed AGNs (Panessa et al. 2006), along with low-mass type 1 AGNs (Greene \& Ho 2007a; Desroches et al. 2009; Dong et al. 2012), low-mass type 2 AGNs (Thornton et al. 2009), and the two low-mass type 1 archetypes NGC 4395 (Panessa et al. 2006) and POX 52 (Thornton et al. 2008). For consistency with the J1231+1106 measurement, all literature $\mathrm{X}$-ray luminosities have been adjusted to the $0.5-2 \mathrm{keV}$ band using published photon indices whenever available or assuming $\Gamma=1.8$ when not. Unexpectedly, the ratio of X-ray to [O III] luminosity in $\mathrm{J} 1231+1106$ - optically a type 2 source-lies securely within the range occupied by unabsorbed type 1 AGNs, both of high and low mass. By contrast, the four low-mass type 2 systems studied by Thornton et al. (2009) all sit substantially below the bulk of the type 1 AGNs in Figure 3, consistent with the X-rays being significantly absorbed in these systems.

Taken at face value, the lack of X-ray absorption in an ostensibly type 2 Seyfert violates the conventional geometric unification picture for AGNs (Antonucci 1993). In this sense J1231+1106 joins a small number of AGNs that seem to intrinsically lack a broad-line region (e.g., Ghosh et al. 2007; Brightman \& Nandra 2011; Tran et al. 2011; Matt et al. 2012). Could the apparent absence of broad lines be an artifact? Probably not. If the $\mathrm{BH}$ mass is as low as $\sim 10^{5} M_{\odot}$ (see below), we expect any broad $\mathrm{H} \alpha$, if present, to be inherently weak and challenging to detect. Our chances of seeing it, however, could have been compromised by the relatively low signal-to-noise ratio of the $\mathrm{H} \alpha$ portion of the spectrum. To estimate an upper limit to the amount of broad $\mathrm{H} \alpha$ allowed by the data, we assume that the hypothetical feature has the median line width of the sample of low-mass type 1 sources from Greene \& Ho (2007c). Given $M_{\mathrm{BH}}=10^{5} M_{\odot}$ and FWHM $=900 \mathrm{~km} \mathrm{~s}^{-1}$, the $\mathrm{H} \alpha$-based virial mass estimator of Greene \& Ho (2005b; as updated in Xiao et al. 2011) predicts $L_{\mathrm{H} \alpha} \approx 5 \times 10^{40} \mathrm{erg} \mathrm{s}^{-1}$, roughly 4 times the observed luminosity of narrow $\mathrm{H} \alpha$. Simple experimentation with the data shows 


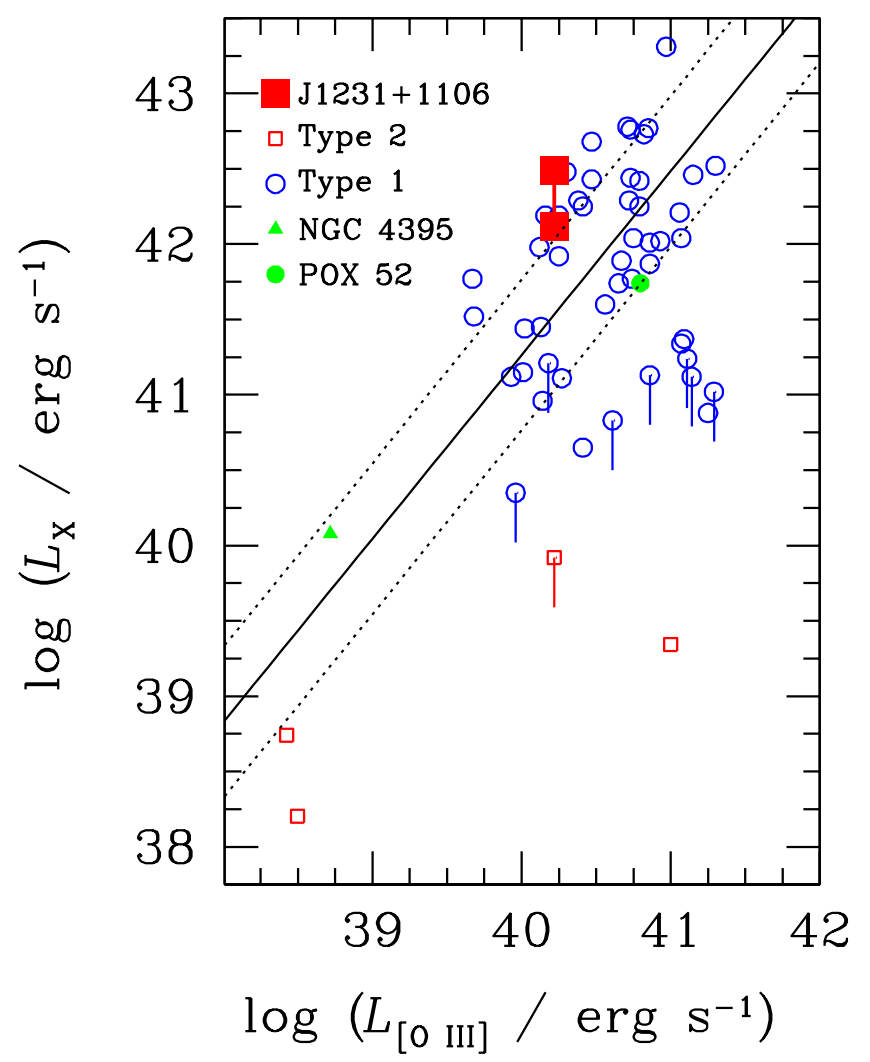

FIG. 3.- Correlation between absorption-corrected soft X-ray luminosity in the $0.5-2 \mathrm{keV}$ band and [O III] $\lambda 5007$ luminosity for J1231+1106 (two large, filled red squares, representing the highest and lowest flux values; Terashima et al. 2012), low-mass type 2 AGNs (open red squares; Thornton et al. 2009); low-mass type 1 AGNs (open blue circles; Greene \& Ho 2007a; Desroches et al. 2009; Dong et al. 2012), NGC 4395 (filled green triangle; Panessa et al. 2006, adjusted to a distance of $4.3 \mathrm{Mpc}$ ), and POX 52 (solid green circle; Thornton et al. 2008). The solid line is the best-fitting relation from Panessa et al. (2006), with the $1 \sigma$ scatter denoted by the two dotted lines; their X-ray luminosities were transformed from the $2-10 \mathrm{keV}$ band to the $0.5-2 \mathrm{keV}$ band assuming $\Gamma=1.8$.

that it is difficult to hide a broad $\mathrm{H} \alpha$ line of this strength, leading us to conclude that $1231+1106$ is genuinely a type 2 source.

As with the type 2 sources studied by Barth et al. (2008) and Thornton et al. (2009), we anticipate that the BH mass in J1231+1106 must be very low. Several aspects of the optical data presented in this study lend themselves to this interpretation. First, the host galaxy is physically small $\left(R_{e} \approx\right.$ $0.7 \mathrm{kpc}$ ), disk-dominated (Sérsic index $n \approx 1$ ), low-luminosity $\left(M_{g}=-17.9 \mathrm{mag}\right.$, corrected to the revised distance and for a Galactic extinction of $A_{g}=0.111 \mathrm{mag}$ ), and, judging by the narrowness of its emission lines $\left(\sigma=33.5 \mathrm{~km} \mathrm{~s}^{-1}\right)$, presumably low-mass. For comparison, J1231+1106 is at least 2 magnitudes fainter than typical field galaxies at $z=0.1$, which have a characteristic luminosity of $M_{g}^{*}=-20.1 \mathrm{mag}$ (Blanton et al. 2003), and over a magnitude fainter than the hosts of most previously studied low-mass type $1\left(\left\langle M_{g}\right\rangle=-19.3 \mathrm{mag}\right.$; Greene \& Ho 2007c) or type $2\left(\left\langle M_{g}\right\rangle=-19.0\right.$ mag; Barth et al. 2008) AGNs. The location of $\mathrm{J} 1231+1106$ on the $[\mathrm{O}$ III] $\lambda 5007 / \mathrm{H} \beta$ vs. [N II] $\lambda 6583 / \mathrm{H} \alpha$ diagnostic diagram additionally hints that the gas-phase metallicity may be sub-solar. These gross properties remind us of bright dwarf galaxies or very late-type spirals. They also share strong morphological similarities with the host galaxies of low-mass type 1 AGNs such as NGC 4395 and many of the Greene \& Ho (2004, 2007c) objects (e.g., Jiang et al. 2011). Curiously, however, J1231+1106 has exceptionally red optical colors $(u-g=1.53 \mathrm{mag} ; g-r=0.83 \mathrm{mag})$ for its luminosity and presumed late morphological type. Late-type spirals have $g-r \lesssim 0.5 \mathrm{mag}$, and even S0 galaxies are seldom redder than $g-r \approx 0.7$ mag (Fukugita et al. 1995). If the host of J1231+1106 is, in fact, disk-dominated, it must be mildly reddened by dust.

The current set of optical observations places an independent constraint on the BH mass and Eddington ratio of J1231+1106. The lack of detectable broad permitted lines prevents us from estimating the mass of the central BH using conventional methods developed for type 1 AGNs. However, we can offer an educated guess using the width of the narrow lines. The velocity dispersion of the narrow-line region gas in AGNs generally traces the gravitational potential of the stars in the host, such that, to first order, $\sigma_{g} \approx \sigma_{*}$. This rough approximation holds for a wide range of AGN types and activity levels (Greene \& Ho 2005a; Ho 2009), including low-mass systems (Barth et al. 2008; Xiao et al. 2011). Moreover, the $M_{\mathrm{BH}}-\sigma_{*}$ relation extends across a wide range of masses, including $M_{\mathrm{BH}} \lesssim 10^{6} M_{\odot}$ (Barth et al. 2005; Greene \& Ho 2006; Xiao et al. 2011). Adopting $\sigma_{*} \simeq \sigma_{g}=33.5 \mathrm{~km} \mathrm{~s}^{-1}$, the $M_{\mathrm{BH}}-\sigma_{*}$ of Gültekin et al. (2009) for inactive galaxies predicts $M_{\mathrm{BH}}=6.8 \times 10^{4} M_{\odot}$. Active galaxies, especially toward lower masses, may define a shallower $M_{\mathrm{BH}}-\sigma_{*}$ relation. The fit of Xiao et al. (2011) yields a slightly larger mass of $M_{\mathrm{BH}}=1.3 \times 10^{5} M_{\odot}$. Although the $M_{\mathrm{BH}}-\sigma_{*}$ relation at these low masses is highly uncertain (Xiao et al. 2011 estimate a formal intrinsic scatter of $0.46 \mathrm{dex}$ ), we can safely conclude that the $\mathrm{BH}$ powering J1231+1106 may be as low as $M_{\mathrm{BH}} \approx 10^{5} M_{\odot}$. Such a value would be in accord with the general characteristics of the host (discussed above), as well as the arguments based on the X-ray properties mentioned in the Introduction.

Following the methodology of Greene \& Ho (2007b), the extinction-corrected $\mathrm{H} \alpha$ luminosity of $L_{\mathrm{H} \alpha}=1.32 \times 10^{40} \mathrm{erg} \mathrm{s}^{-1}$ (Section 3) implies $L_{\mathrm{bol}}=2.34 \times 10^{44}\left(L_{\mathrm{H} \alpha} / 10^{42}\right)^{0.86}=5.66 \times$ $10^{42} \mathrm{erg} \mathrm{s}^{-1}$, or $L_{\mathrm{bol}} / L_{\mathrm{Edd}}=0.45$ for $M_{\mathrm{BH}}=10^{5} M_{\odot}$. If we adopt the lower value of $M_{\mathrm{BH}}=6.8 \times 10^{4} M_{\odot}, L_{\mathrm{bol}} / L_{\mathrm{Edd}}=0.66$. Either of these two values is in excellent agreement with the expectation that the Eddington ratio should exceed $\sim 0.3$ to account for the observed Comptonization of the X-ray spectrum (Terashima et al. 2012).

Lastly, we computed a few simple photoionization models using the code CLOUDY (Ferland et al. 1998) to test whether the unusual X-ray spectrum of J1231+1106 can be responsible for the excitation of its optical lines. We assume that the ionizing continuum can be described by a blackbody with $T=1.7 \times 10^{6} \mathrm{~K}$, corresponding to a $0.15 \mathrm{keV}$ blackbody that can approximate the $\mathrm{X}$-ray spectrum. We vary the ionization parameter in the range $U=10^{-4.5}-10^{-1.5}$ and the hydrogen density $n_{\mathrm{H}}=10^{2}-10^{6} \mathrm{~cm}^{-3}$, values typical of the narrow-line regions of most AGNs (e.g., Nagao et al. 2001; Kim et al. 2006). For simplicity we assume solar metallicity, plane-parallel geometry, and constant hydrogen density and neglect the effects of dust. These calculations confirm that the observed X-ray spectral energy distribution of J1231+1106 can, indeed, reproduce line ratios that span the locus of AGNs in standard diagnostic diagrams such as that shown in Figure 2. Given the simplicity of the models, we did not attempt to fine-tune them to exactly match $\mathrm{J} 1231+1106$. 
This work is supported by the Carnegie Institution for Science (LCH), a KASI-Carnegie Fellowship (MK), and Grantin-Aid for Scientific Research 20740109 (YT) from the Min- istry of Education, Culture, Sports, Science, and Technology of Japan.

\section{REFERENCES}

Abazajian, K., Adelman-McCarthy, J. K., Agüeros, M. A., et al. 2009, ApJS, 182,543

Ai, Y. L., Yuan, W., Zhou, H. Y., Wang, T. G., \& Zhang, S. H. 2011, ApJ, 727, 31

Antonucci, R. 1993, ARA\&A, 31, 473

Araya Salvo, C., Mathur, S., Ghosh, H., Fiore, F., \& Ferrarese, L. 2012, ApJ, 757,179

Baldwin, J. A., Phillips, M. M., \& Terlevich, R. 1981, PASP, 93, 5

Barth, A. J., Greene, J. E., \& Ho, L. C. 2005, ApJ, 619, L151

Barth, A. J., Greene, J. E., \& Ho, L. C. 2008, AJ, 136, 1179

Barth, A. J., Ho, L. C., Rutledge, R. E., \& Sargent, W. L. W. 2004, ApJ, 607, 90

Blanton, M. R., Hogg, D. W., Bahcall, N. A., et al. 2003, ApJ, 592, 819

Brightman, M., \& Nandra, K. 2011, MNRAS, 414, 3084

Cardelli, J. A., Clayton, G. C., \& Mathis, J. S. 1989, ApJ, 345, 245

Desroches, L.-B., Greene, J. E., \& Ho, L. C. 2009, ApJ, 698, 1515

Desroches, L.-B., \& Ho, L. C. 2009, ApJ, 690, 267

Dong, R. B., Greene, J. E., \& Ho, L. C. 2012, ApJ, submitted

Ferland, G. J., Korista, K. T., Verner, D. A., et al. 1998, PASP, 110, 761

Filippenko, A. V., \& Ho, L. C. 2003, ApJ, 588, L13

Fukugita, M., Shimasaku, K., \& Ichikawa, T. 1995, PASP, 107, 945

Ghosh, H., Pogge, R. W., Mathur, S., Martini, P., \& Shields, J. C. 2007, ApJ, 656,105

Greene, J. E., \& Ho, L. C. 2004, ApJ, 610, 722

Greene, J. E., \& Ho, L. C. 2005a, ApJ, 627, 721

Greene, J. E., \& Ho, L. C. 2005b, ApJ, 630, 122

Greene, J. E., \& Ho, L. C. 2006, ApJ, 641, L21

Greene, J. E., \& Ho, L. C. 2007a, ApJ, 656, 84

Greene, J. E., \& Ho, L. C. 2007b, ApJ, 667, 131

Greene, J. E., \& Ho, L. C. 2007c, ApJ, 670, 92

Gültekin, K., Richstone, D. O., Gebhardt, K., et al. 2009, ApJ, 698, 198
Halpern, J. P., \& Steiner, J. E. 1983, ApJ, 269, L37

Ho, L. C. 2009, ApJ, 699, 638

Ho, L. C., \& Kim, M. 2009, ApJS, 184, 398

Jiang, Y.-F., Greene, J. E., Ho, L. C., Xiao, T., \& Barth, A. J. 2011, ApJ, 742, 68

Kamizasa, N., Terashima, Y., \& Awaki, H. 2012, ApJ, 751, 39

Kauffmann, G., Heckman, T. M., Tremonti, C., et al. 2003, MNRAS, 346, 1055

Kewley, L. J., Groves, B., Kauffmann, G., \& Heckman, T. 2006, MNRAS, 372, 961

Kim, M., Ho, L. C. \& Im, M. 2006, ApJ, 642, 702

Ludwig, R. R., Greene, J. E., Barth, A. J., \& Ho, L. C. 2012, ApJ, 756, 51

Matt, G., Bianchi, S., Guainazzi, M., Barcons, X., \& Panessa, F. 2012, A\&A, 540, A111

Miniutti, G., Ponti, G., Greene, J. E., et al. 2009, MNRAS, 394, 443

Nagao, T., Murayama, T., \& Taniguchi, Y. 2001, ApJ, 546, 744

Panessa, F., Bassani, L., Cappi, M., et al. 2006, A\&A, 455, 173

Papadakis, I. E. 2004, MNRAS, 348, 207

Peng, C. Y., Ho, L. C., Impey, C. D., \& Rix, H.-W. 2010, AJ, 139, 2097

Satyapal, S., Böker, T., Mcalpine, W., et al. 2009, ApJ, 704, 439

Schlafly, E. F., \& Finkbeiner, D. P. 2011, ApJ, 737, 103

Sérsic, J. L. 1968, Atlas de Galaxias Australes (Córdoba: Obs. Astron., Univ. Nac. Córdoba)

Terashima, Y., Kamizasa, N., Awaki, H., Kubota, A., \& Ueda, Y. 2012, ApJ, 752,154

Thornton, C. E., Barth, A. J., Ho, L. C., \& Greene, J. E. 2009, ApJ, 705, 1196

Thornton, C. E., Barth, A. J., Ho, L. C., Rutledge, R. E., \& Greene, J. E. 2008, ApJ, 686, 892

Tran, H. D., Lyke, J. E., \& Mader, J. A. 2011, ApJ, 726, L21

Vaughan, S., Iwasawa, K., Fabian, A. C., \& Hayashida, K. 2005, MNRAS, 356, 524

Xiao, T., Barth, A. J., Greene, J. E., et al. 2011, ApJ, 739, 28 